\title{
Surgical rheumatic mitral valve repair compared with percutaneous balloon mitral valvuloplasty in mitral stenosis in current era: a propensity score matching study
}

\author{
Jie Han ${ }^{1 \#}$, Baiyu Tian ${ }^{1 \#}$, Fang $\mathrm{Wu}^{1 \#}$, Yuqing Jiao ${ }^{1}$, Shuai Pang ${ }^{2}$, Jinguo $\mathrm{Xu}^{3}, \mathrm{Xu} \mathrm{Meng}^{1}$ \\ ${ }^{1}$ Cardiac Valve Center, Department of Cardiac Surgery, Beijing Anzhen Hospital, Capital Medical University-Beijing Institute of Heart Lung and \\ Blood Vessel Diseases, Beijing, China; ${ }^{2}$ Center of Cardiovascular Surgery, the People's Hospital of Huaiyin Jinan, Jinan, China; ${ }^{3}$ Department of \\ Cardiovascular Surgery, the First Affiliated Hospital of Anhui Medical University, Hefei, China \\ Contributions: (I) Conception and design: J Han, B Tian, X Meng; (II) Administrative support: X Meng; (III) Provision of study materials or patients: J \\ Han, F Wu; (IV) Collection and assembly of data: B Tian, S Pang, Y Jiao; (V) Data analysis and interpretation: B Tian, J Han, F Wu; (VI) Manuscript \\ writing: All authors; (VII) Final approval of manuscript: All authors. \\ "These authors contributed equally to this work. \\ Correspondence to: Xu Meng. Cardiac Valve Center, Department of Cardiac Surgery, Beijing Anzhen Hospital, Capital Medical University, No. 2 \\ Anzhen Road, Beijing, China. Email: mxu_az@126.com.
}

Background: Many comparative studies of percutaneous balloon mitral valvuloplasty (PBMV) and surgical mitral commissurotomy (SMC) in rheumatic mitral stenosis (MS) were done in the last few decades. With the development of valve repair techniques, various surgical rheumatic valve repair techniques have been applied in clinic, but there is a lack of comparison with PBMV. Our study was designed to compare the perioperative and mid-term outcomes of PBMV and mitral valve repair with "four-step" procedure in the treatment of rheumatic MS.

Methods: Patients with MS were treated with PBMV or rheumatic mitral valve repair (rMVP) at Beijing Anzhen Hospital between January 1, 2013 and September 30, 2018 were selected. By using propensity score matching (PSM) method, we compared the changes in post-operation clinical outcomes between the two matched groups. Kaplan-Meier analyses was used for survival analysis and drawing the curve, and log-rank test were used to compare intergroup differences.

Results: A total of 252 cases were enrolled after selection, 74 cases in PBMV and 178 cases in rMVP. Seventy-four pairs were matched successfully after PSM. There were 53 females in PBMV and 54 in rMVP. The mean age of two groups was $46.95 \pm 12.50$ and $47.55 \pm 11.91$ years respectively. There was no significantly differences in mitral valve orifice area (MVOA) $\left(1.05 \pm 0.32\right.$ vs. $\left.0.97 \pm 0.24 \mathrm{~cm}^{2}, \mathrm{P}=0.12\right)$ and left ventricular ejection fraction (EF) $(62.36 \% \pm 5.17 \%$ vs. $62.52 \% \pm 4.94 \%, \mathrm{P}=0.76)$ between two groups preoperatively. Baseline characteristics were basically balanced after PSM. In each group, there was one case transferred to surgical mitral valve replacement due to the failure of valvuloplasty before discharge. All patients survived the interventions and no severe complications were found. MVOA were significantly increased in rMVP compared with PBMV postoperatively, as well as grading of MS and tricuspid regurgitation (TR) were significantly improved in rMVP. Three cases in PBMV were lost during the follow-up. Mitral replacement was performed in 11 patients and one of them died in PBMV, while none of patients underwent reintervention in rMVP, but one patient died of pneumonia.

Conclusions: For selected patients with rheumatic MS in China, our study shows that there are comparable clinical outcomes in terms of operative, mid-term mortality and complications between PBMV and surgical rMVP with "four-step" procedure. Surgical rMVP shows more advantageous in the correction of valve stenosis and the management of concomitant tricuspid valve lesions and atrial fibrillation (AF).

Keywords: Rheumatic valve disease; mitral stenosis (MS); rheumatic mitral repair; percutaneous balloon mitral valvuloplasty (PBMV); propensity score matching (PSM) 
Submitted Apr 17, 2020. Accepted for publication Sep 23, 2020.

doi: $10.21037 /$ jtd-20-1694

View this article at: http://dx.doi.org/10.21037/jtd-20-1694

\section{Introduction}

Great changes have taken place in the treatment for rheumatic mitral stenosis (MS) in the recent few decades. Early on Henry and Bailey used their fingers to dilate the mitral valve orifice $(1,2)$. After that, as the development in extracorporeal circulation technique, the mitral valve morphology could be clearly seen by the surgeon during the surgery, which promoted the surgical mitral commissurotomy (SMC) $(3,4)$. Percutaneous balloon mitral valvuloplasty (PBMV) (5) was applied in clinic in 1984, which greatly changed the treatment strategy of rheumatic MS since it was less invasive than surgery and could also solve the problem of MS.

Mitral valve repair has been proved to be the best surgical treatment for patients with degenerative mitral regurgitation (MR) and it is popularized in the world. The safety and effectiveness of rheumatic mitral valve repair (rMVP) have been confirmed in many studies $(6,7)$. However, since rheumatic inflammation always involves the whole mitral apparatus, repair is often complex and requires a combination of a variety of repair techniques. The lack of simple and effective rheumatic mitral repair procedure makes it difficult to be widely used at present. Thus, PBMV is still the preferred treatment for rheumatic MS according to the European and American guidelines for heart valve disease $(8,9)$, which is based on the uncertainty outcomes of surgical rMVP. Furthermore, the main surgical treatment of rheumatic mitral valve is still prosthetic valve replacement. In the view of the decreased cardiac function caused by valve replacement, the emergence of anticoagulation-related complications, and the decline of long-term survival $(10,11)$, PBMV may provide an opportunity for patients, especially for young patients, to delay valve replacement. On the other hand, considering that compared with surgery, PBMV is less invasive and less dangerous, patients' choice will be more inclined to PBMV.

Rheumatic mitral valve disease is a major heart valvular disease in China. Although the current morbidity has been greatly reduced with the development of health care in China, there are still lots of patients suffer from rheumatic valvular disease. In the past decade, as cardiopulmonary bypass and surgical valve surgery techniques matures and common in China, the application of PBMV has gradually decreased and surgical valve replacement is more general. With the innovation of rMVP technology, our center has carried out rMVP since 2013-“four-step" procedurewhich is mainly based on commissuroplasty, and achieved good results.

To evaluate clinical differences between PBMV and mitral valve repair, we conducted a retrospective study on patients with rheumatic MS.

We present the following article in accordance with the STROBE reporting checklist (available at http://dx.doi. org/10.21037/jtd-20-1694).

\section{Methods}

\section{Patients selection}

From January 2013 to September 2018, patients who underwent PBMV at cardiology center or underwent surgical rMVP at heart valvular center of Beijing Anzhen Hospital were selected. Exclusion criteria included concomitant significant MR, aortic valve procedures or coronary artery bypass grafting operations and previous cardiac surgeries. Concomitant tricuspid valve repair or atrial radio-frequency ablation was not excluded. Seventyfour cases of PBMV and 178 cases of rMVP were enrolled after selection. Seventy-four pairs were successfully matched after propensity score matching (PSM) method and reviewed.

\section{Procedure}

\section{PBMV}

Inoue balloon technique was performed by experienced physicians in the catheterization laboratory. Hemodynamic variables were obtained before and after each balloon inflation.

\section{rMVP}

rMVP in our center was performed with a "four-step" procedure in which commissuroplasty as a main operative technique, as describe by Luo et al. (12). Patients with atrial fibrillation (AF) underwent Cox-maze IV procedure and 
left atrial appendage (LAA) was closed from inside of left atrial (LA) simultaneously. Tricuspid valve repair was based on the evaluation by echocardiography and the observation intraoperation.

\section{Echocardiography evaluation}

Comprehensive two-dimensional and color Doppler echocardiographic evaluation was performed in all patients before and after PBMV or rMVP. routine measurements of cardiac chamber dimensions and ejection fraction were calculated. Based on 2014 and 2017 AHA/ACC Guidelines for the Management of Patients With Valvular Heart Disease, the degree of MS was graded as: very severe MS, [mitral valve orifice area (MVOA): $<1.0 \mathrm{~cm}^{2}$ ), severe MS (MVOA: $1.0-1.5 \mathrm{~cm}^{2}$ ); progressive MS (MVOA: $1.5-2.0 \mathrm{~cm}^{2}$ ); the degree of MR was graded as mild [effective regurgitant orifice (ERO): $<0.2 \mathrm{~cm}^{2}$ ], moderate (ERO: $0.2-0.4 \mathrm{~cm}^{2}$ ) or severe (ERO: $\geq 0.4 \mathrm{~cm}^{2}$ ).

\section{Follow-up}

Clinical follow-up data were collected until March 2020 by telephone interviews, e-mail or follow-up website of our center. Clinical data included survival, re-intervention, New York Heart Association (NYHA) function and complications. The main end points were defined as the clinical events of death and re-intervention during follow-up.

\section{Statistical analysis}

Clinical and echocardiographic variables were collected to be analyzed via SPSS Statistic Version 22.0 for Windows (IBM Corp., Armonk, NY, USA). Continuous variables were presented as a mean \pm SD and were compared by the Student $t$-tests or the non-parametric Mann-Whitney $\mathrm{U}$ tests. Categorical variables are presented as numbers and percentages and were compared by Fisher's exact test.

Since there were imbalanced cases between the two groups in which less PBMV were done than rMVP, there might have been inevitable bias at the baseline characteristics between these two groups. A PSM study therefore was introduced for analysis. We picked out gender, age, LA diameter, left ventricular end-diastolic diameter (LVEDD), left ventricular end-systolic diameter (LVESD), left ventricular ejection fraction (EF), MVOA, MS degree and NYHA as covariables, 0.2 as the caliper width and chose a 1:1 match to adjust cases of the two groups in order to reduce the bias. By comparing the data and drawing a survival curve after PSM, a difference of prognosis between the two groups was observed and discussed.

Kaplan-Meier analysis was used to determine survival rate and events-free rate, as well as Kaplan-Meier curves are shown for the time-to-event distributions of the main end point. The log-rank test was used to examine the differences between the two groups. A $\mathrm{P}$ value lower than 0.05 was considered to indicate significance.

All patients in this study were provided informed consent for undergoing the procedures. The study was conducted in accordance with the Declaration of Helsinki (as revised in 2013). The study design was approved by the Ethics Review Committee of Beijing Anzhen Hospital, Capital Medical University, Beijing, China (No. 2019096x).

\section{Results}

\section{Population description}

From January 2013 to September 2018, a total of 252 patients were collected, comprising $74 \mathrm{PBMV}$ patients and 178 rMVP patients before PSM. The mean age was $46.96 \pm 12.5$ (range, 24-78) years and 50.31 \pm 12.07 (range, 25-74) years respectively. Baseline clinical and echocardiographic characteristics pre- and post-PSM are summarized in Table 1. There were statistical differences in age, gender, NYHA function, LA diameter, mitral valve effective orifice area, and AF between the two groups at the baseline before PSM. Patients in rMVP had a higher age, a higher class of NYHA function, and a higher proportion of patients with tricuspid insufficiency and AF compared with patients in PBMV. The mean MOVA was smaller in PBMV $\left(0.97 \pm 0.24\right.$ vs. $\left.1.13 \pm 0.29 \mathrm{~cm}^{2}, \mathrm{P}<0.01\right)$, but there was no significant difference in MS degree distribution.

The differences of baseline characteristics between the two groups were basically eliminated after PSM (Figure 1). No statistical differences were found in echocardiographic characteristics. However, there was still significant difference in TR between two groups (Table 1).

\section{Early results}

All of the 148 patients survived the interventions. Each group had one patient receiving mitral valve replacement before discharge because of severe MR intra-operation. Postoperative echocardiography data are summarized in Table 2. The mean size of balloon in PBMV was 
Table 1 Baseline characteristics of the patients in two groups pre- vs. post-PSM

\begin{tabular}{|c|c|c|c|c|c|c|}
\hline Characteristics & \multicolumn{3}{|c|}{ Pre-PSM } & \multicolumn{3}{|c|}{ Post-PSM } \\
\hline Age, years & $46.95 \pm 12.50$ & $50.31 \pm 12.07$ & 0.04 & $46.95 \pm 12.50$ & $47.55 \pm 11.91$ & 0.76 \\
\hline Gender, female & 53 & 148 & 0.04 & 53 & 54 & 0.85 \\
\hline NYHA & & & $<0.01$ & & & 0.07 \\
\hline III & 22 & 101 & & 22 & 30 & \\
\hline IV & 4 & 5 & & 4 & 0 & \\
\hline LA, mm & $44.60 \pm 8.86$ & $48.64 \pm 8.54$ & $<0.01$ & $44.60 \pm 8.86$ & $45.95 \pm 7.32$ & 0.39 \\
\hline LVEDD, mm & $44.40 \pm 3.81$ & $46.44 \pm 4.80$ & $<0.01$ & $44.40 \pm 3.81$ & $44.74 \pm 4.15$ & 0.65 \\
\hline $\mathrm{MVOA}, \mathrm{cm}^{2}$ & $0.97 \pm 0.24$ & $1.13 \pm 0.29$ & $<0.01$ & $0.97 \pm 0.24$ & $1.05 \pm 0.32$ & 0.12 \\
\hline Emax, $\mathrm{cm} / \mathrm{s}$ & $215.62 \pm 46.58$ & $190.82 \pm 58.36$ & $<0.01$ & $215.62 \pm 46.58$ & $214.20 \pm 65.06$ & 0.93 \\
\hline MS & & & 0.36 & & & 0.18 \\
\hline Moderate & $1(1.4)$ & $8(4.5)$ & & $1(1.4)$ & $5(6.8)$ & \\
\hline Severe & $28(37.8)$ & 74 (41.6) & & 28 (37.8) & $22(29.7)$ & \\
\hline Very severe & $45(60.8)$ & $96(53.9)$ & & $45(60.8)$ & 47 (63.5) & \\
\hline TR & & & $<0.01$ & & & 0.04 \\
\hline None/mild & $53(71.6)$ & $104(58.4)$ & & $53(71.7)$ & $45(61.1)$ & \\
\hline
\end{tabular}

$26 \mathrm{~mm}$ and the mean size of annuloplasty ring was 32 $\mathrm{mm}$ in rMVP. MVOA was significantly larger in $\mathrm{rMVP}$ compared with PBMV $\left(2.12 \pm 0.33\right.$ vs. $1.64 \pm 0.29 \mathrm{~cm}^{2}$, $\mathrm{P}<0.01$ ) (Figure 2), as well as the MS degree was more improved in $\mathrm{rMVP}(\mathrm{P}<0.01)$. In addition, $\mathrm{TR}$ was also decreased obviously in $\mathrm{rMVP}(\mathrm{P}=0.03)$.

\section{Follow-up outcomes}

We examined a mid-term results of the adjusted patients. The median follow-up was 43.72 months (total 3,104 months) in the PBMV group and 44.5 months (total 3,293 months) in the rMVP group. Three patients were lost during follow-up in PBMV (95.9\% complete) and no patients were lost in rMVP (100\% complete).

Mitral valve replacement was performed in 11 patients in the group PBMV during the follow-up, 7 for restenosis and 4 for regurgitation. One of them died from the operation. No re-operation was performed in patients in the rMVP group, but 1 patient died from pneumonia 

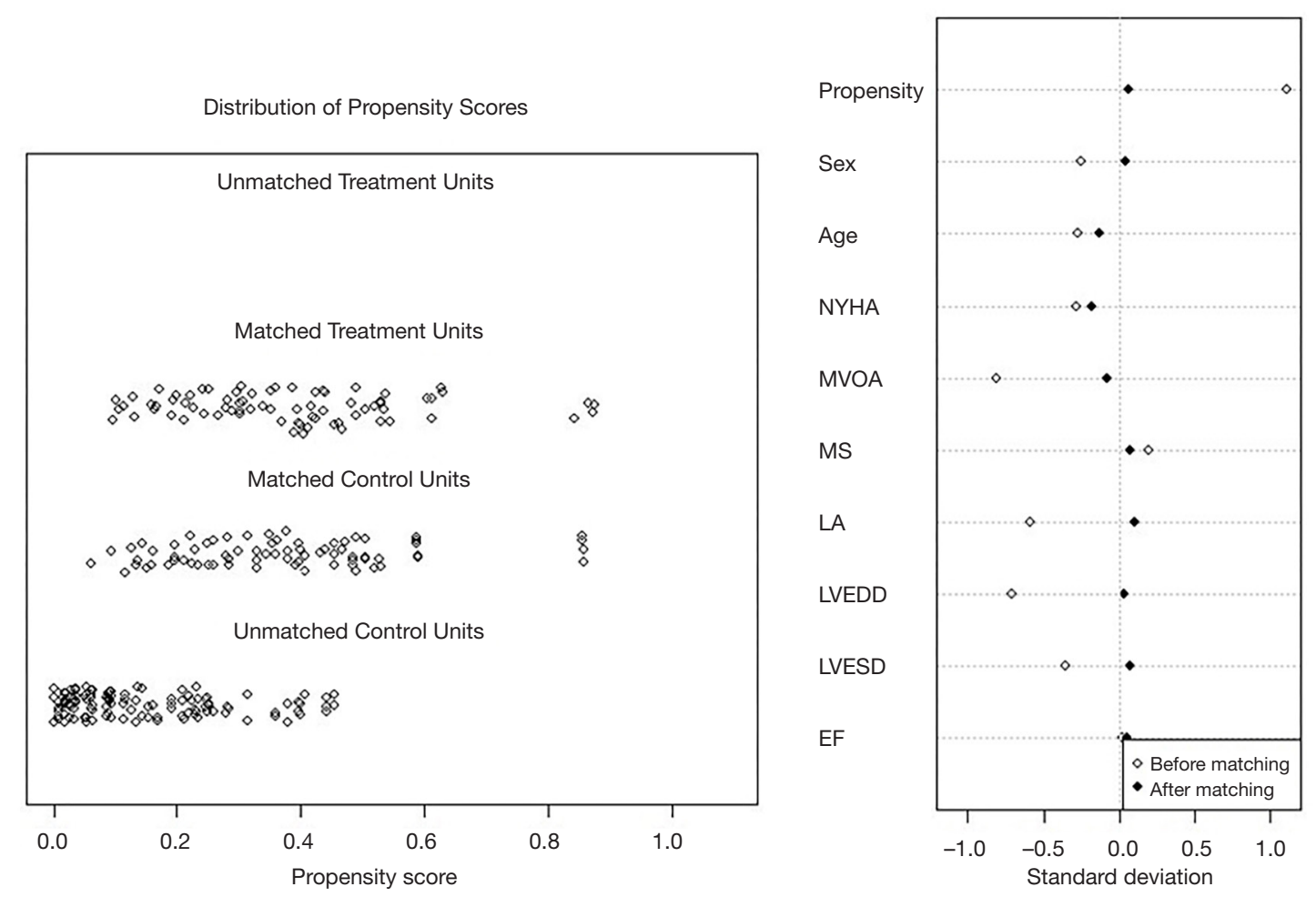

Figure 1 Distribution of propensity scores and standard deviation pre- and post-PSM. Treatment represent PBMV group and Control represent rMVP group. The scatter diagram of standard deviation shows the standardized difference of each covariates before and after matching. Standard deviation, which is between $0.0 \pm 0.10$, indicating that the variable is in equilibrium after matching. Y-axis shows covariates, the number of $\mathrm{X}$-axis means standard deviation. PSM, propensity score matching; PBMV, percutaneous balloon mitral valvuloplasty; rMVP, rheumatic mitral valve repair; NYHA, New York Heart Association; MVOA, mitral valve orifice area; MS, mitral stenosis; LA, left atrial; LVEDD, left ventricular end-diastolic diameter; LVESD, left ventricular end-systolic diameter; EF, left ventricular ejection fraction.

infection. All patients without re-intervention achieved a functional improvement of NYHA classification. KaplanMeier survival curve analysis showed that there was a higher risk of re-intervention at 7 years in PBMV compared with rMVP $(98.65 \% \pm 1.34 \%$ vs. $72.62 \% \pm 9.37 \%, \mathrm{P}=0.002)$ (Figure 3), but there was no significant difference in survival between the groups (Figure 4).

\section{Discussion}

Chronic rheumatic valvular heart disease is the most common valvular disease in developing countries. As the most public chronic rheumatic valvular heart disease, mitral valve stenosis threats people's health seriously. PBMV was regarded as a new-type treatment for a long time in the past, and many comparative studies on PBMV and surgery open or closed commissurotomy were reported to confirm the efficiency of PBMV (13-15).

With the deepening understanding of mitral valve anatomy, mitral valve repair technique gradually developed more than merely divide the adherent tissue. Different from the traditional commissurotomy concept, current rheumatic mitral repair not only deals with the adhesive commissure caused by rheumatic inflammation, but also needs to correct the lesion of leaflets, subvalvular apparatus and the annulus. Many cardiac surgeons around the world had been trying to repair rheumatic mitral valve, which was sometimes difficult, but the feasibility, safety, and effectiveness of rheumatic mitral repair were well demonstrated $(6,7,16,17)$.

The results of existing studies on PBMV, which mostly preceded in the end of last century, showed that MVOA was 1.6 to $2.0 \mathrm{~cm}^{2}(13,18-21)$ post-PBMV. Data from our research indicated that both PBMV and rMVP can improve MVOA, but the improvement in $\mathrm{MVP}$ was 
Table 2 Postoperative echocardiography variables

\begin{tabular}{|c|c|c|c|c|c|c|}
\hline Variables & \multicolumn{3}{|c|}{ Pre-PSM } & \multicolumn{3}{|c|}{ Post-PSM } \\
\hline $\mathrm{LA}, \mathrm{mm}$ & $43.19 \pm 6.19$ & $44.48 \pm 8.75$ & 0.37 & $43.19 \pm 6.19$ & $43.62 \pm 5.95$ & 0.71 \\
\hline LVEDD, mm & $44.26 \pm 7.17$ & $45.12 \pm 4.31$ & 0.31 & $44.26 \pm 7.17$ & $45.44 \pm 4.24$ & 0.27 \\
\hline LVESD, mm & $28.88 \pm 3.85$ & $28.78 \pm 2.86$ & 0.85 & $28.88 \pm 3.85$ & $28.92 \pm 2.90$ & 0.96 \\
\hline MVOA, $\mathrm{cm}^{2}$ & $1.67 \pm 0.26$ & $2.05 \pm 0.34$ & $<0.01$ & $1.67 \pm 0.26$ & $2.12 \pm 0.33$ & $<0.01$ \\
\hline Emax, $\mathrm{cm} / \mathrm{s}$ & $162.57 \pm 39.98$ & $160.11 \pm 32.87$ & 0.66 & $162.57 \pm 39.98$ & $159.38 \pm 30.95$ & 0.65 \\
\hline MS & & & $<0.01$ & & & $<0.01$ \\
\hline Moderate & $42(57.5)$ & $82(46.9)$ & & $42(57.5)$ & $30(41.1)$ & \\
\hline TR & & & $<0.01$ & & & 0.03 \\
\hline None/mild & $62(84.9)$ & 166 (94.9) & & $62(84.9)$ & 70 (95.9) & \\
\hline Moderate & $5(6.8)$ & $8(4.6)$ & & $5(6.8)$ & $3(4.1)$ & \\
\hline Severe & $6(8.3)$ & $1(0.5)$ & & $6(8.3)$ & 0 & \\
\hline
\end{tabular}

Data are presented as mean \pm SD or $n$ (\%). PSM, propensity score matching; PBMV, percutaneous balloon mitral valvuloplasty; rMVP, rheumatic mitral valve repair; LA, left atrial; LVEDD, left ventricular end-diastolic diameter; LVESD, left ventricular end-systolic diameter; EF, left ventricular ejection fraction; MVOA, mitral valve orifice area; Emax, transmitral E peak velocity; MS, mitral stenosis; TR, tricuspid regurgitation.

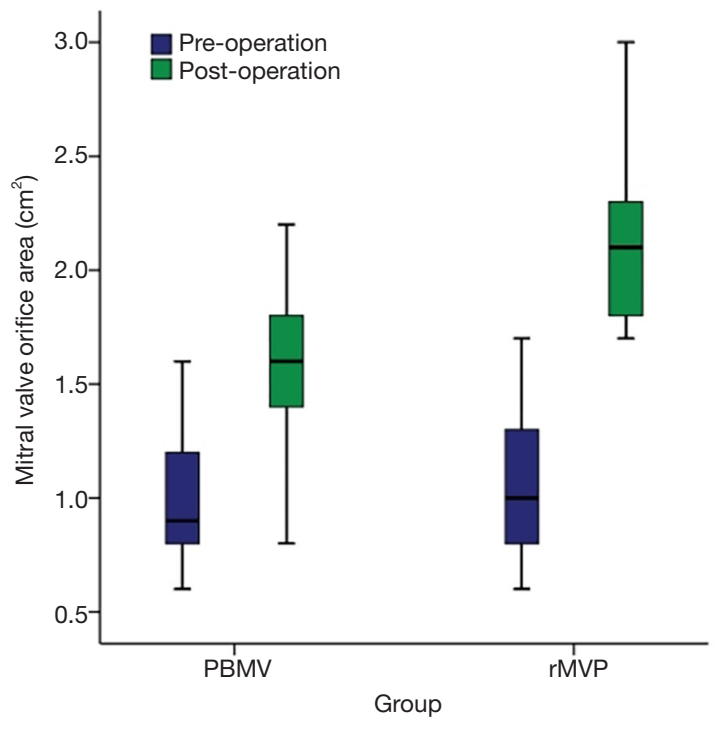

Figure 2 Preoperative and postoperative changes in MVOA of two groups. MVOA was significantly improved postoperatively in both groups, but more obviously in rMVP. MVOA, mitral valve orifice area; rMVP, rheumatic mitral valve repair; PBMV, percutaneous balloon mitral valvuloplasty.

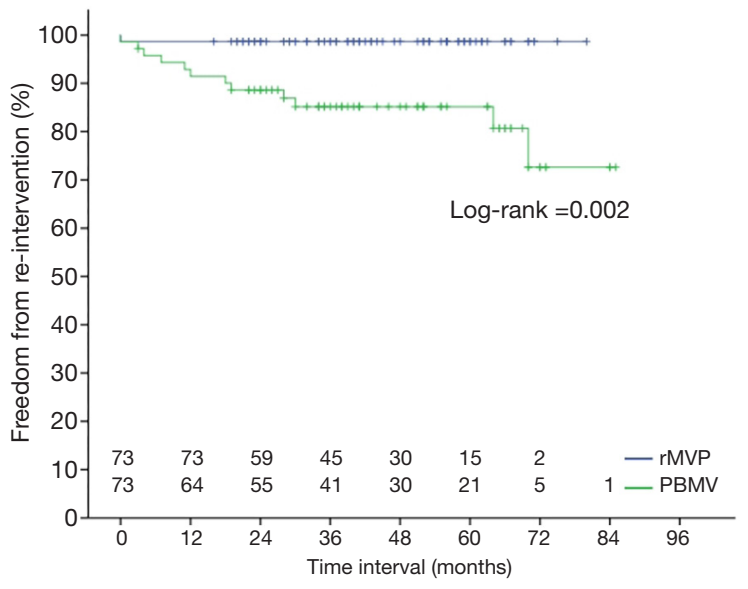

Figure 3 Freedom from re-intervention in the two groups. Re-intervention in the group PBMV was significantly higher than that in group rMVP $(\mathrm{P}=0.002)$. PBMV, percutaneous balloon mitral valvuloplasty; rMVP, rheumatic mitral valve repair. 


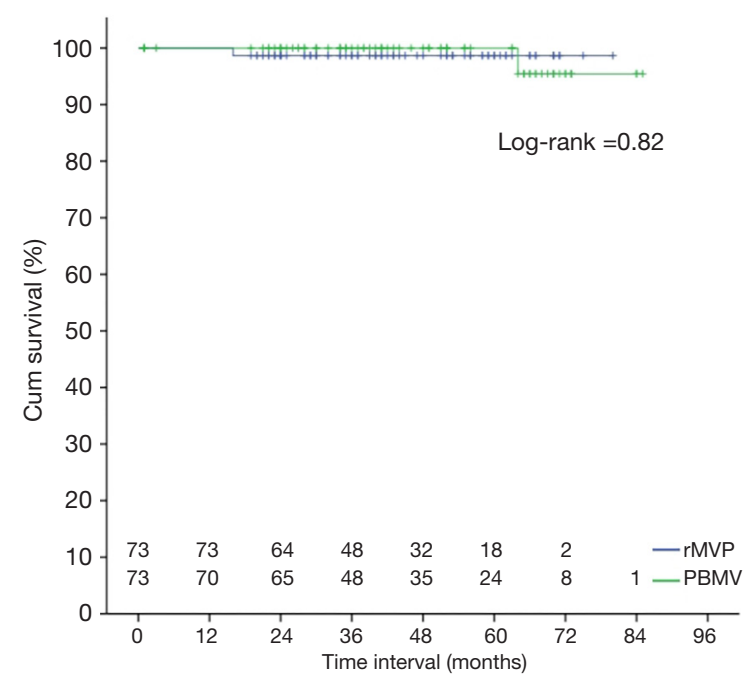

Figure 4 Survival in the group PBMV compared with group rMVP. There was no significant difference between groups ( $\mathrm{P}=0.82)$. PBMV, percutaneous balloon mitral valvuloplasty; rMVP, rheumatic mitral valve repair.

greater than PBMV $\left(2.12 \pm 0.33\right.$ vs. $\left.1.67 \pm 0.26 \mathrm{~cm}^{2}, \mathrm{P}<0.01\right)$. Although there were significant differences in ejection fraction between the groups, which considered to be caused by extracorporeal circulation's affection, they were still in the normal interval. Serious MR is treated as a risk of procedure failure in both PBMV and rMVP, with a chance of $1.4 \%$ to $9.4 \%(22,23)$ and $2.6 \%$ to $3.2 \%(12,24)$ respectively. However, whether PBMV or rMVP could be used on a patient or not based on multiple factors, including the patient's condition, their choice, and the surgeon's skill. Therefore, the evaluation of echocardiography preoperation and the morphology of mitral valve intraoperation is essential (25). One patient from both PBMV and rMVP in our study failed the repair procedure because the instant echocardiography showed serious MR after procedure; However, the success rate had no significant difference between PBMV and rMVP.

Previous research showed that the re-intervention rate of $\mathrm{PBMV}$ in 5 years is $2 \%$ to $26 \%$ (26); while the re-operation rate of rMVP in 10 years is $7.5 \%$ to $13 \%$ (27). Our data showed that patients with $\mathrm{rMVP}$ had a significant lower rate of re-intervention than patients with PBMV. This result is similar with the report of $\mathrm{Hu}$ et al. (28), in which the reintervention rate of $\mathrm{PBMV}$ is significantly higher than SMC (RR: 2.88, 95\% CI: 1.97-4.20; $\mathrm{P}<0.01$ ).

Mitral valve repair is based on the function of mitral apparatus, which not only deals with the commissure, but also remove calcified or fibered tissue as well as loose the adhesive chordae or papillary muscle, and in this way restore the motion of the valve. Furthermore, with the application of artificial annuloplasty ring, the natural mitral annulus can be stabilized and coaptation height can be increased as well. $\mathrm{PBMV}$ is only based on opening the narrow mitral valve by dilatation. Because the expansion position is uncontrollable during the process, such "blind tearing" tends to damage the weakest part of the mitral valve rather than the planned anatomical position. As a result, normal structure is destroyed and surgical repair would be hardly operated after PBMV. Therefore, the two procedures lead to different effect after interventions.

In present study, one patient in PBMV died because of left ventricular rupture 64 months after surgical valve replacement; and another 69-year patient in rMVP died because of lung infection 16 months after surgery. There was no significant difference of mortality between two procedures. At present, the safety of cardiac surgery in China has been greatly improved with the development of health care compared with the last two decades and the long-term complication rate and mortality of surgical repair were significantly lower than that of replacement. Although balloon valvuloplasty is available for some restenosis PBMV patients, most of them require surgery in China. However, because of prior damage of the mitral valve is often difficult to repair, valve replacement is chosen instead, which potentially increases correlative risks.

Certainly, for certain distinct patients, such as pregnancy, PBMV have obvious benefit. The safety of PBMV on pregnant patients has been confirmed (29,30). In this study, there were eight pregnant patients in the PBMV group, with a median gestational week of 27 and an interval of 9 to 28 weeks. Procedures were done successfully in all patients and subsequent delivery smoothly; all infants were thoroughly unaffected. Compared to the risk of surgery, such as anesthetic drugs, cardiopulmonary bypass, and blood loss, whose fetal mortality is $30 \%$ to $40 \%$ and maternal mortality is $9 \%(9,30,31)$, the risk of PBMV exactly is much lower.

\section{Limitation}

The current study has several limitations inherent to the retrospective analysis, data used to evaluate valve calcification was missing, Wilkins score in PBMV group were not documented in electronic medical record system, 
for example. Also, as the choice of treatment depends on the department which the patient visited, there was bias in selection of intervention strategy. Because of the development in valve surgery in China, more patients were treated with mitral valve surgery instead of PBMV, resulting in a relatively low number of cases. In order to overcome these effects, we used PSM to balance the baseline characteristics of the patients in two groups. Lack of data regarding follow-up echocardiography, which could affect evaluate the valve structure, is another limitation of this study. Moreover, taking into consideration the life expectancy, cost-benefit and other complications is required. Hence, further studies are needed.

\section{Conclusions}

For selected patients with rheumatic MS in China, our study shows that there are comparable clinical outcomes in terms of operative, mid-term mortality and complications between PBMV and surgical rheumatic valve repair with "four-step" procedure. Surgical rMVP shows more advantageous in the correction of valve stenosis with a lower incidence of mid-term re-intervention and the management of concomitant tricuspid valve lesions and $\mathrm{AF}$.

\section{Acknowledgments}

Funding: None.

\section{Footnote}

Reporting Checklist: The authors have completed the STROBE reporting checklist. Available at http://dx.doi. org/10.21037/jtd-20-1694

Data Sharing Statement: Available at http://dx.doi. org/10.21037/jtd-20-1694

Conflicts of Interest: All authors have completed the ICMJE uniform disclosure form (available at http://dx.doi. org/10.21037/jtd-20-1694). The authors have no conflicts of interest to declare.

Ethical Statement: The authors are accountable for all aspects of the work in ensuring that questions related to the accuracy or integrity of any part of the work are appropriately investigated and resolved. All patients in this study were provided informed consent for undergoing the procedures. The study was conducted in accordance with the Declaration of Helsinki (as revised in 2013). The study design was approved by the Ethics Review Committee of Beijing Anzhen Hospital (No. 2019096x).

Open Access Statement: This is an Open Access article distributed in accordance with the Creative Commons Attribution-NonCommercial-NoDerivs 4.0 International License (CC BY-NC-ND 4.0), which permits the noncommercial replication and distribution of the article with the strict proviso that no changes or edits are made and the original work is properly cited (including links to both the formal publication through the relevant DOI and the license). See: https://creativecommons.org/licenses/by-nc-nd/4.0/.

\section{References}

1. Bailey CP. The surgical treatment of mitral stenosis (mitral commissurotomy). Dis Chest 1949;15:377-97.

2. Harken DE, Ellis LB, et al. The surgical treatment of mitral stenosis; valvuloplasty. N Engl J Med 1948;239:801-9.

3. Durán CG, Revuelta JM, Gaite L, et al. Stability of mitral reconstructive surgery at 10-12 years for predominantly rheumatic valvular disease. Circulation 1988;78:191-6.

4. Scalia D, Rizzoli G, Campanile F, et al. Long-term results of mitral commissurotomy. J Thorac Cardiovasc Surg 1993;105:633-42.

5. Inoue K, Owaki T, Nakamura T, et al. Clinical application of transvenous mitral commissurotomy by a new balloon catheter. J Thorac Cardiovasc Surg 1984;87:394-402.

6. Pomerantzeff PM, Brandao CM, Leite Filho OA, et al. Mitral valve repair in rheumatic patients with mitral insuficiency: twenty years of techniques and results. Rev Bras Cir Cardiovasc 2009;24:485-9.

7. Thomas MP, Badhwar V. A three-step technique for repair of rheumatic disease of the mitral valve. Cardiol Young 2014;24:1104-7.

8. Baumgartner H, Falk V, Bax JJ, et al. 2017 ESC/EACTS Guidelines for the management of valvular heart disease. Eur Heart J 2017;38:2739-91.

9. Nishimura RA, Otto CM, Bonow RO, et al. 2014 AHA/ ACC guideline for the management of patients with valvular heart disease: a report of the American College of Cardiology/American Heart Association Task Force on Practice Guidelines. J Am Coll Cardiol 2014;63:e57-185.

10. Dillon J, Yakub MA, Kong PK, et al. Comparative longterm results of mitral valve repair in adults with chronic 
rheumatic disease and degenerative disease: is repair for "burnt-out" rheumatic disease still inferior to repair for degenerative disease in the current era? J Thorac Cardiovasc Surg 2015;149:771-7; discussion 777-9.

11. McNeely CA, Vassileva CM. Long-term outcomes of mitral valve repair versus replacement for degenerative disease: a systematic review. Curr Cardiol Rev 2015;11:157-62.

12. Luo T, Meng X, Yan Z, et al. Commissuroplasty as a main operative technique in rheumatic mitral valve repair: surgical experiences and mid-term results. Heart Lung Circ 2020;29:940-8.

13. Cotrufo M, Renzulli A, Ismeno G, et al. Percutaneous mitral commissurotomy versus open mitral commissurotomy: a comparative study. Eur J Cardiothorac Surg 1999;15:646-51; discussion 651-2.

14. Ommen SR, Nishimura RA, Grill DE, et al. Comparison of long-term results of percutaneous mitral balloon valvotomy with closed transventricular mitral commissurotomy at a single North American Institution. Am J Cardiol 1999;84:575-7.

15. Rifaie O, Abdel-Dayem MK, Ramzy A, et al. Percutaneous mitral valvotomy versus closed surgical commissurotomy. Up to 15 years of follow-up of a prospective randomized study. J Cardiol 2009;53:28-34.

16. Chan PG, Hayanga AJ, Badhwar V. Repair of rheumatic mitral stenosis with bicommissural release, anterior leaflet augmentation and oversized annuloplasty. Multimed Man Cardiothorac Surg 2014;2014:mmt020.

17. Chotivatanapong T, Lerdsomboon P, Sungkahapong V. Rheumatic mitral valve repair: experience of 221 cases from Central Chest Institute of Thailand. J Med Assoc Thai 2012;95 Suppl 8:S51-7.

18. Dean LS, Mickel M, Bonan R, et al. Four-year followup of patients undergoing percutaneous balloon mitral commissurotomy. A report from then national heart, lung, and blood institute balloon valvuloplasty registry. J Am Coll Cardiol 1996;28:1452-7.

19. Nobuyoshi M, Arita T, Shirai S, et al. Percutaneous balloon mitral valvuloplasty: a review. Circulation 2009;119:e211-9.

20. Saccocci M, Taramasso M, Maisano F. Percutaneous mitral valvuloplasty in the modern era. Kardiol Pol 2018;76:819-20.

21. Song JK, Kim MJ, Yun SC, et al. Long-term outcomes of percutaneous mitral balloon valvuloplasty versus open cardiac surgery. J Thorac Cardiovasc Surg 2010;139:103-10.

22. Chen CR, Cheng TO. Percutaneous balloon mitral valvuloplasty by the Inoue technique: a multicenter study of 4832 patients in China. Am Heart J 1995;129:1197-203.

23. Palacios IF, Sanchez PL, Harrell LC, et al. Which patients benefit from percutaneous mitral balloon valvuloplasty? Prevalvuloplasty and postvalvuloplasty variables that predict long-term outcome. Circulation 2002;105:1465-71.

24. Bakir I, Onan B, Onan IS, et al. Is rheumatic mitral valve repair still a feasible alternative?: indications, technique, and results. Tex Heart Inst J 2013;40:163-9.

25. Wilkins GT, Southern JF, Choong CY, et al. Correlation between echocardiographic endocardial surface mapping of abnormal wall motion and pathologic infarct size in autopsied hearts. Circulation 1988;77:978-87.

26. Yazicioğlu N, Arat Özkan A, Orta Kiliçkesmez K, et al. Immediate and follow-up results of repeat percutaneous mitral balloon commissurotomy for restenosis after a succesful first procedure. Echocardiography 2010;27:765-9.

27. Mihos CG, Pineda AM, Capoulade R, et al. A systematic review of mitral valve repair with autologous pericardial leaflet augmentation for rheumatic mitral regurgitation. Ann Thorac Surg 2016;102:1400-5.

28. Hu X, Zhao Q. Systematic comparison of the effectiveness of percutaneous mitral balloon valvotomy with surgical mitral commissurotomy. Swiss Med Wkly 2011;141:w13180.

29. Brigui M, Remadi F, Belkhiria N, et al. Results of percutaneous mitral dilatation in 11 cases of poorly tolerated mitral stenosis during pregnancy. Ann Cardiol Angeiol (Paris) 1994;43:129-34.

30. de Souza JAM, Martinez EE, Ambrose JA, et al. Percutaneous balloon mitral valvuloplasty in comparison with open mitral valve commissurotomy for mitral stenosis during pregnancy. J Am Coll Cardiol 2001;37:900-3.

31. Sliwa K, Johnson MR, Zilla P, et al. Management of valvular disease in pregnancy: a global perspective. Eur Heart J 2015;36:1078-89.

Cite this article as: Han J, Tian B, Wu F, Jiao Y, Pang S, Xu J, Meng X. Surgical rheumatic mitral valve repair compared with percutaneous balloon mitral valvuloplasty in mitral stenosis in current era: a propensity score matching study. J Thorac Dis 2020;12(11):6752-6760. doi: 10.21037/jtd-20-1694 